\title{
Increasing the performance of a system by changing reliability
}

\author{
Claudiu Babiș ${ }^{1}$, Andrei Dimitrescu ${ }^{1, *}$,Ana Maria Alecusan², and Marcela Babiș ${ }^{3}$ \\ ${ }^{1}$ University Politehnica of Bucharest, Faculty of Industrial Engineering and Robotics, Bucharest, \\ Romania \\ ${ }^{2}$ Goethe German College, Bucharest, Romania \\ ${ }^{3}$ Secondary School no.1, Berceni, Romania
}

\begin{abstract}
The evaluation of the performance of a mechanical system takes into account many factors, including those related to competitiveness, technology and reliability.

From the point of view of competitiveness, most companies use the same state-of-the-art information obtained either by applying their own research, development and innovation resources, or by purchasing technologies and patents from other companies or from the free market. Thus the resulting products, if not innovative products, are part of the same range.

The global technological level has reached a very high degree of development, using unconventional technologies with high precision, robots, advanced mechanical systems and assembly lines with high productivity as well as verification and control systems on manufacturing flow which makes production to be uniform.

Differences between manufacturers begin to appear when the degree of customer satisfaction changes, which is also found in the concept of reliability. Thus, the one who will find the technological solution with the same number of parts, but with a high reliability, will win the market. The paper aims at a detailed study with direct reference to how to increase reliability at the design stage.
\end{abstract}

\section{Introducere}

The operational safety of industrial equipment, devices and components has led to the emergence of the concept of reliability, and is now an indispensable technique for engineers. [1]

In the reliability design stage, a very important element is the harmonization of the values of the three main types of reliability: projected reliability (preliminary, predicted) which represents the reliability based on the design concept or on the knowledge of the reliability of its components under prescribed operating conditions; experimental (technical) reliability which represents the experimentally established reliability in specialized laboratories, test stations, test stands, in which the behavior of the product is monitored by simulating the

\footnotetext{
*Corresponding author: andrei_dimitrescu@yahoo.com
} 
operating conditions, and the operational (operational) reliability which represents the reliability of the product received by the beneficiary.[9]

Reliability is one of the essential elements of the quality of a product as shown in Figure $1[4]$

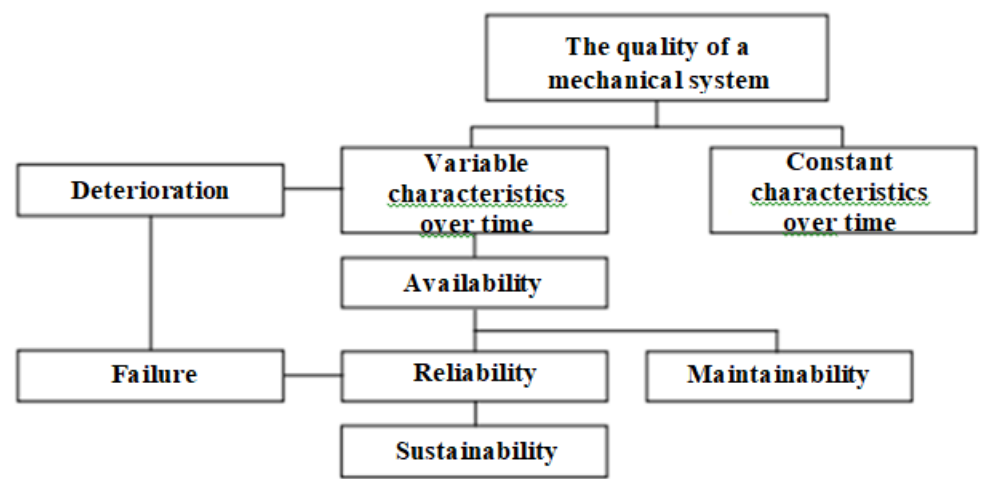

Fig.1. The place of reliability within the concept of quality.[4]

Quantitatively, the reliability is the characteristic of a product expressed by the probability of fulfilling the imposed function for a given time $T_{f}$ longer than the time interval L, prescribed, in specific operating conditions, meaning: [2]

$$
R(L)=\mathrm{P}\left(T_{\mathrm{f}}>L\right)
$$

The optimal variant from a technological point of view is the correct determination of the reliability values of each element that is part of the system. This can be done in two ways: by consulting the literature and the manufacturers who supply these benchmarks or, if they are a novelty in the consumer market, by their own tests. [10] The second variant has a number of advantages, but also disadvantages. Disadvantages include additional production costs, more time to determine reliability, and the main advantages are the possibility of improving values by changing operating parameters and placing new products on the market.

In order to prevent catastrophic defects, it is necessary to introduce the notion of deterioration in the concept of reliability. Deterioration is the process of gradually changing the constructive and functional parameters on which the correct operation of the system depends. In the case of mechanical systems, the phenomenon of deterioration consists in deformations, ruptures, leaks, material changes. The failure occurs when the limit value of one of the deterioration processes affecting the system is reached as shown in Figure 2. [3]

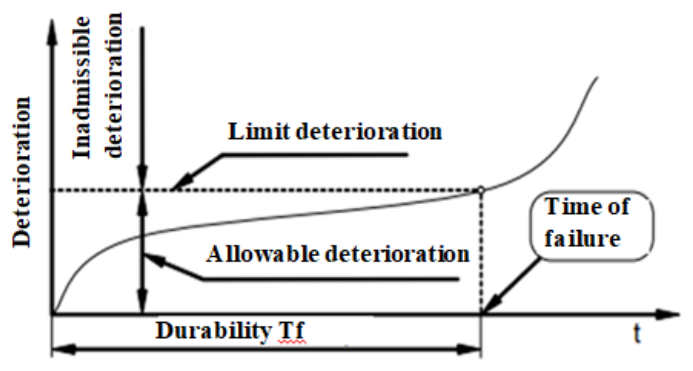

Fig. 2. Connection between deterioration and failure.[3]

In the case of certain processes and systems, the deterioration may be expressed as a percentage. Exceeding these values does not cause the actual failure, but impermissibly 
increases the risk of its occurrence, implicitly changing the reliability. Also, for certain important mechanical systems, the occurrence of the failure can be predicted by the periodic assessment of the degree of damage.

It is known from theory that using several elements arranged in parallel decreases the probability of system failure and therefore increases the probability of proper functioning.[7]

The present paper aims at a study through which, using the same components of the system, by organizing them in parallel type, superior values of reliability can be obtained compared to the classic series type.

This principle can be widely applied in various industries, through a stage of improving design from the point of view of reliability engineering.

\section{The Analyzed System}

In the industrial practice of heating systems it has been found that over $62 \%$ of the failures of the series installations are produced due to the overload of the first heating system. Usually, this failure leads to chain stresses on the other heating systems and finally to the general shutdown.

The study underlying the research presented in this paper is a heating system of an industrial space with an area of $480 \mathrm{~m}^{2}$ and a volume of $1920 \mathrm{~m}^{3}$ which is part of an energy class with the following performances:

Table 1. Energy performance of the building.

\begin{tabular}{|c|c|c|}
\hline Characteristic & Value $\left[\mathrm{kWh} / \mathrm{m}^{2} \mathrm{an}\right]$ & Energy performance \\
\hline Heating & 180 & $\mathrm{D}$ \\
\hline Hot water consumption & - & $\mathrm{C}$ \\
\hline Lighting & 52 & - \\
\hline Conditioning & - & $\mathrm{D}$ \\
\hline Mechanical ventilation & 11 & D \\
\hline TOTAL & $\mathbf{2 4 3}$ & \\
\hline
\end{tabular}

Thus, in order to obtain an optimal temperature for the development of activities, two heating systems have been designed that use two heating units with a nominal power of 52 $\mathrm{kW}$.

The first system has the two units connected in series (figure 3), and the second system has the units connected in parallel (figure 4).

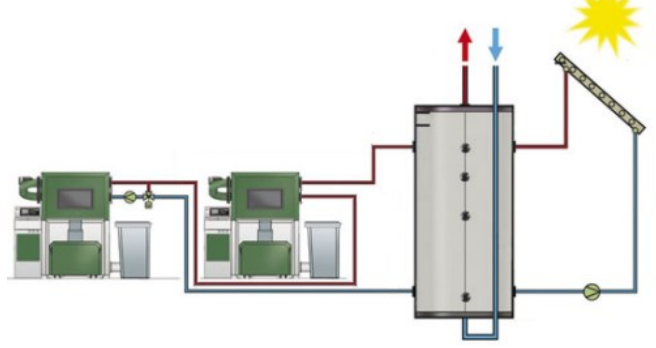

Fig. 3. Heating system mounted in series. 
It can be seen that this heating system is a simple one in which the heating of the water, which is stored in the buffer tank, is done by passing hot water that leaves the first unit and enters the second. The system is monitored by a controller that changes the operating parameters in real time.

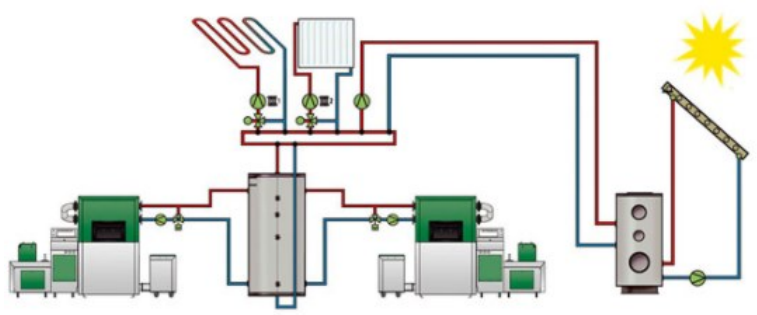

Fig. 4. Heating system with units mounted in parallel.

Heating systems with units mounted in parallel are the optimal option from several points of view: economical - because the power required is evenly distributed, so energy consumption is lower; technological - because both power plants are worn evenly, and by installing intelligent control units and when the power requirement is low, it will use the two boilers in turn so that the operating time is equal; reliability - use of boilers for equal periods of time and request of all components within the parameters prescribed by the manufacturer.

Also, the situations in which the use of these series boiler systems is recommended are those in which the nominal energy power of the installation is high. The disadvantages of using a single boiler instead of the above-mentioned system are multiple. For example, from the point of view of energy consumption, a $120 \mathrm{~kW}$ boiler with a maximum thermal heating power of $60-80^{\circ} \mathrm{C}$ of $110,5 \mathrm{~kW}$ has a consumption of $11,90 \mathrm{~m}^{3} / \mathrm{h}$ of natural gas, and two units of $54 \mathrm{~kW}$ each with a maximum thermal power at heating $60-80^{\circ} \mathrm{C}$ of $52,9 \mathrm{~kW}$ each has a cumulative consumption of $9,72 \mathrm{~m}^{3} / \mathrm{h}$ of natural gas.

\section{Reliability calculation in the design stage}

In order for the project beneficiary to obtain the highest possible quality of the whole set, the reliability of each system must be calculated and all the advantages and disadvantages that it offers must be analyzed.

As the domestic hot water supply is not of interest to the beneficiary, the solar panel heating system can be eliminated from both heating variants.

For ease of calculation, 268 simple elements and subsystems were removed, resulting in 8 elements for effective reliability calculation. It should also be noted that in the case of the first design variant a BKU type cascade controller was used, thus increasing the degree of automation of the installation.

By consulting the specialized literature, as well as the dedicated equipment suppliers, the following values of reliability were obtained:[5]

Table 2. Reliability values of system components.

\begin{tabular}{|c|c|c|}
\hline Characteristic code & Characteristic & Value [\%] \\
\hline 1 & Control unit & 74 \\
\hline 2 & Waterfall regulator & 82 \\
\hline 3 & Cold water supply system & 92 \\
\hline
\end{tabular}




\begin{tabular}{|c|c|c|}
\hline 4 & Central heating (1-2) & 65 \\
\hline 5 & Three-way valve & 75 \\
\hline 6 & AT flow temperature sensor & 88 \\
\hline 7 & Exterior temperature sensor & 70 \\
\hline 8 & Circulation pump & 90 \\
\hline
\end{tabular}

The next step is to design the heating system with two units mounted in series (figure 5)

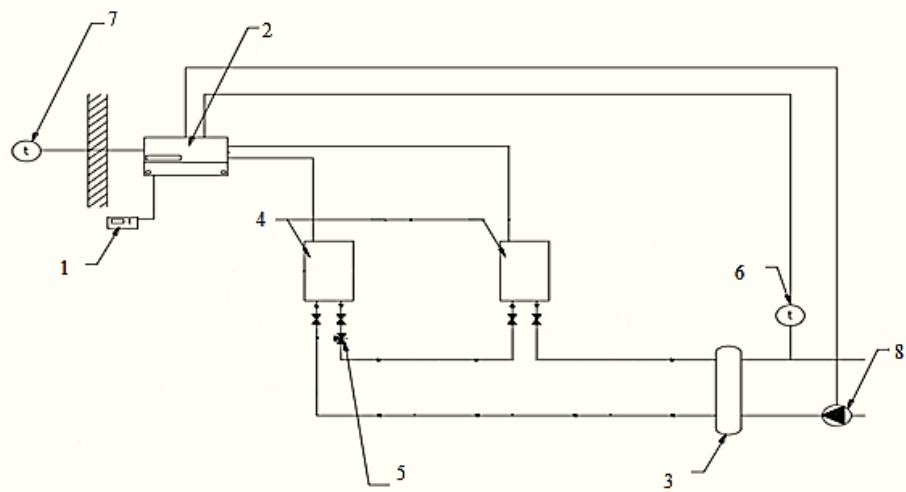

Fig. 5. Diagram of the heating system with units mounted in series: 1- control unit, 2 - BKU cascade controller, 3 - cold water supply system, 4 -heating units, 5 - three-way valve, 6 - AT temperature sensor - flow , 7 - outdoor temperature sensor, 8 - recirculation pump.

The logic diagram of the reliability of the elements is presented in figure 6

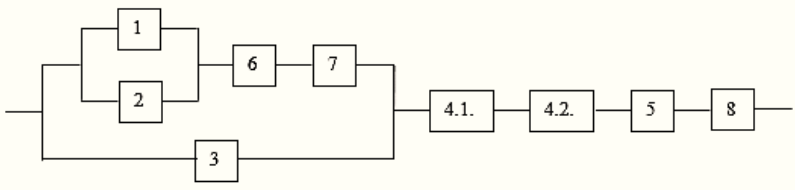

Fig. 6. Logical scheme of operation of the serial system.

$$
R_{1,2,6,7}=\left[1-\left(1-R_{1}\right) \times\left(1-R_{2}\right)\right] \times R_{6} \times R_{7}
$$

By entering the values from table 2 in relation (2) we obtain:

$$
\begin{gathered}
R_{1,2,6,7}=00,587171 \\
R_{1,2,6,7,3}=1-\left(1-R_{1,2,6,7}\right) \times\left(1-R_{3}\right)=0,966974 \\
R_{\text {sistem serie }}=R_{1,2,6,7,3} \times R_{4.1} \times R_{4.2} \times R_{8}=0,275769
\end{gathered}
$$

In the next stage, the heating system with two units mounted in parallel or in a cascade was designed (figure 7) 


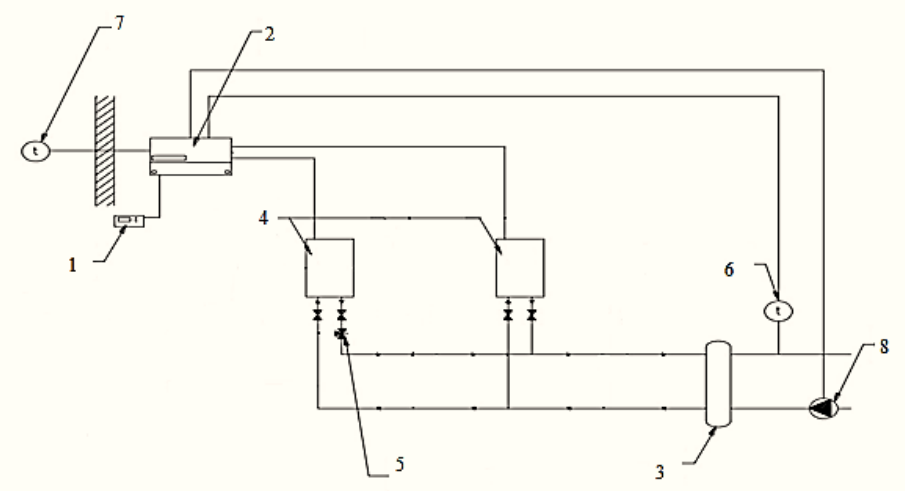

Fig. 7. Diagram of the heating system with the units mounted in parallel: 1 - control unit, 2 - SCU cascade controller, 3 - cold water supply system, 4 - heating units, 5 - three-way valve, 6 - AT temperature sensor - flow , 7 - outdoor temperature sensor, 8 - recirculation pump.

The logic diagram of the cascade system is shown in the figure 8

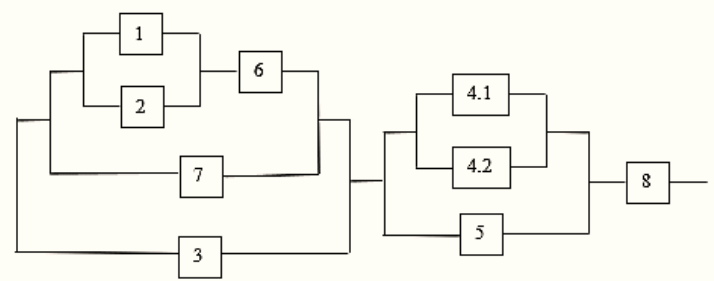

Fig. 8. Logic scheme of operation of the parallel system.

$$
R_{1,2,6}=\left[1-\left(1-R_{1}\right) \times\left(1-R_{2}\right)\right] \times R_{6}
$$

By entering the values from table 2 in relation (6) we obtain:

$$
\begin{gathered}
R_{1,2,6}=0,838816 \\
R_{1,2,6,7}=1-\left(1-R_{1,2,6}\right) \times\left(1-R_{7}\right)=0,951649 \\
R_{1,2,6,7,3}=1-\left(1-R_{1,2,6,7}\right) \times\left(1-R_{3}\right)=0,996132 \\
R_{4.1,4.2}=1-\left(1-R_{4.1}\right) \times\left(1-R_{4.2}\right)=0,8775 \\
R_{4.1,4.2,5}=1-\left(1-R_{4.1,4.2}\right) \times\left(1-R_{5}\right)=0,969375 \\
R_{\text {sistem paralel }}=R_{1,2,6,7,3} \times R_{4.1,4.2,5} \times R_{8}=0,869063
\end{gathered}
$$

\section{Control of the system cascade}

The primary circuit is controlled in three distinct ways: manual control of the operation of each unit; step control via cascade switches; smooth adjustment by the cascade control unit (BKU).

Manual control is performed by setting the operating parameters of each unit, primarily the temperature. This adjustment option requires the constant presence of a person and the reliability of the system cannot be guaranteed as human error is introduced. 
Step control is performed using cascade switches. Controls the system as a set of power steps; when the load changes, the individual units of the cascade start (stop).

The most efficient is the smooth adjustment with the help of the SCU. In this case, the minimum stage of power change is reached. Units must be equipped with modulating burners. Cascade control units can be integrated with room temperature sensors and weatherdependent automation systems.

Connecting heating units in a cascade using a cascade control unit is a complex solution and has a higher efficiency. This block ensures the alternative operation of all heating units and guarantees the same number of operating hours for each heat generator, which maintains the reliability of each subassembly separately at the values generated by the manufacturer. The block optimizes the operation of the system and ensures that only the required number of heat generators are turned on, depending on the required power.

When working with modular burners, the cascade control unit, in addition to the principle described above, seeks to ensure the operation of the heating units in partial power mode (in the power mode in which the unit has the maximum energy efficiency). The most efficient is the use of a cascade control unit together with condensing heating units. In this case, the power provided by the units is most suitable for energy consumption.

It has been confirmed in practice that during the heating season (the period when the authorities allow the centralized distribution of heat), $80 \%$ of the time, the capacity of the heating unit is required only by $50 \%$. That is, for the season as a whole (for one year), the unit is used only at $30 \%$ of maximum power. This means that most of the time it operates at a minimum power with low efficiency.

The low efficiency is directly correlated with the percentage of unit power, as the ratio between energy consumed (natural gas) and energy provided (hot water for radiators) is variable. [11] Each unit manufacturer provides detailed information for the energy ratio. These are usually in form of graphs with data on energy consumption and power offered or energy consumption and the maximum temperature it can generate in the heating medium. Because of this, using a high-power unit is not the most effective solution.

While the cascading scheme provides the necessary power at a time, connecting one after the other, the required number of heating units and bringing them to the optimal economic mode. Cascading, software-controlled regulation eliminates the influence of the imbalance factor on heat generation power and the need for heat from consumers. The cascading power switch allows you to work constantly with a low heating water temperature, which reduces radiation losses during periods of system inactivity.

Cascading boilers is an effective technique for increasing the unit power of a heating device, which has been used by heating specialists for many years. The concept of reception is simple: we divide the total heat load between two or more independently controlled units and include in the cascade only those that satisfy the demand for a given load at a given time. Each unit represents its own "step" of the heating capacity in the total capacity of the system. An intelligent controller (microcontroller) constantly monitors the flow temperature of the heating medium and determines the system steps that must be started to maintain the set temperature.

\section{Collecting experimental data}

For verification, in practice the two systems were created: one in series and the other in parallel (figure 9) using similar components and following the assembly diagram briefly presented in figures 5 and 7. 


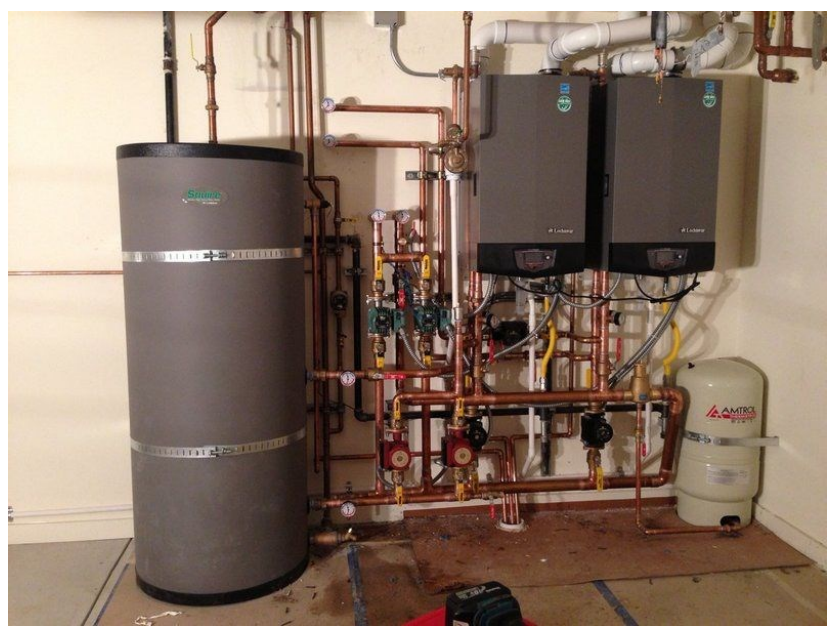

Fig. 9. Parallel system installation.

The falls (failures) of the two systems over 6 periods of time with the same duration were measured. The measured characteristics were the same: 8 subsystems shown in Table 2 .

This resulted in the measurements of the series and parallel systems shown in Tables 3 and 4, as well as the related histograms shown in Figures 10 and 11:

Table 3. Fault values of serial system components.

\begin{tabular}{|l|c|c|c|c|c|c|c|c|}
\hline & rep1 & rep2 & rep3 & rep4 & rep5 & rep6 & rep7 & rep8 \\
\hline I & 2 & 2 & 0 & 5 & 2 & 1 & 2 & 1 \\
\hline II & 3 & 1 & 1 & 3 & 2 & 0 & 1 & 0 \\
\hline III & 1 & 4 & 0 & 7 & 1 & 0 & 1 & 0 \\
\hline IV & 0 & 1 & 0 & 11 & 1 & 1 & 2 & 1 \\
\hline V & 1 & 0 & 1 & 4 & 0 & 1 & 0 & 0 \\
\hline VI & 1 & 1 & 0 & 6 & 1 & 0 & 1 & 1 \\
\hline Mediate & $\mathbf{1 , 3 3}$ & $\mathbf{1 , 5}$ & $\mathbf{0 , 3 3}$ & $\mathbf{6}$ & $\mathbf{1 , 1 6}$ & $\mathbf{0 , 5}$ & $\mathbf{1 , 2}$ & $\mathbf{0 , 5}$ \\
\hline
\end{tabular}

\section{SERIAL SYSTEM}

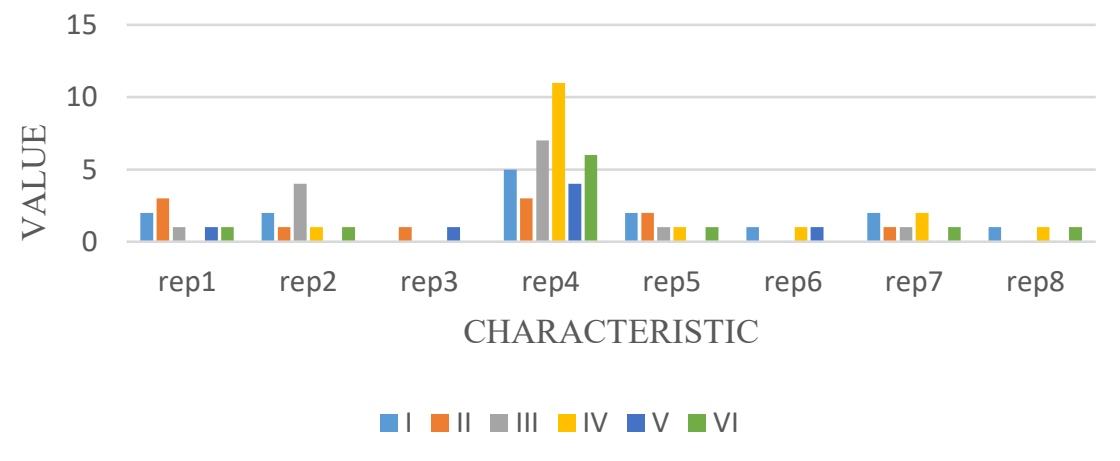

Fig. 10. Histogram of serial system failures. 
Table 4. Fault values of parallel system components.

\begin{tabular}{|l|c|c|c|c|c|c|c|c|}
\hline & rep1 & rep2 & rep3 & rep4 & rep5 & rep6 & rep7 & rep8 \\
\hline I & 2 & 1 & 1 & 2 & 0 & 2 & 0 & 3 \\
\hline II & 2 & 2 & 1 & 1 & 0 & 0 & 1 & 0 \\
\hline III & 0 & 1 & 0 & 3 & 1 & 1 & 0 & 1 \\
\hline IV & 0 & 2 & 1 & 1 & 2 & 0 & 2 & 0 \\
\hline V & 2 & 0 & 0 & 1 & 1 & 0 & 0 & 0 \\
\hline VI & 1 & 3 & 1 & 0 & 0 & 2 & 1 & 0 \\
\hline Mediate & $\mathbf{1 , 1 6}$ & $\mathbf{1 , 5}$ & $\mathbf{0 , 6 6}$ & $\mathbf{1 , 3 3}$ & $\mathbf{0 , 6 6}$ & $\mathbf{0 , 8 3}$ & $\mathbf{0 , 6 6}$ & $\mathbf{0 , 6 6}$ \\
\hline
\end{tabular}

PARALLEL SYSTEM

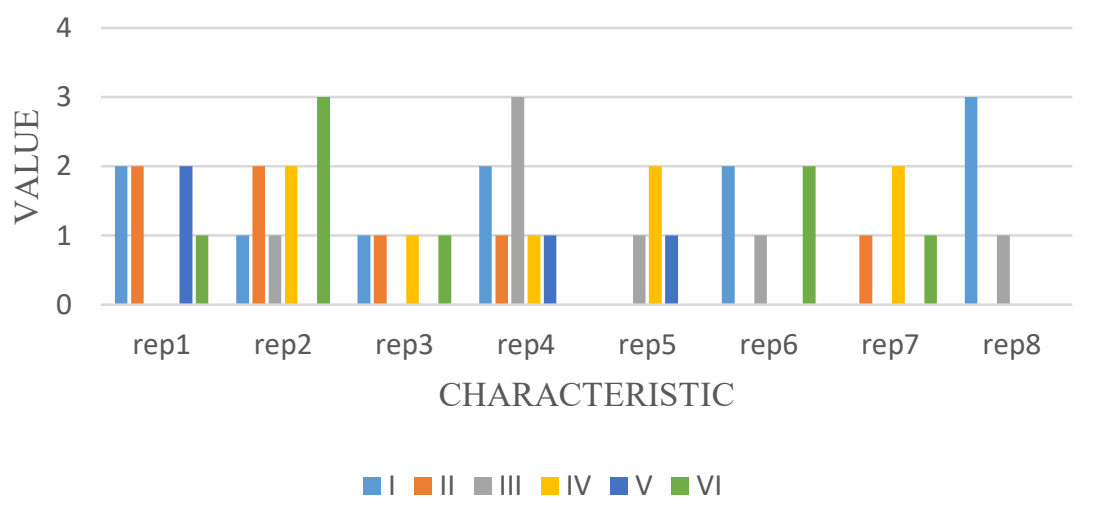

Fig. 11. Parallel system failure histogram.

In order to better observe the two systems, we made a comparative presentation, averaging the values of the failures of each component for the studied period, thus resulting in table 5 and figure 12:

Table 5. Mediated values of failures of serial and parallel system components.

\begin{tabular}{|l|c|c|c|c|c|c|c|c|}
\hline & rep1 & rep2 & rep3 & rep4 & rep5 & rep6 & rep7 & rep8 \\
\hline Mediate Serial System & 1,33 & 1,5 & 0,33 & 6 & 1,16 & 0,5 & 1,16 & 0,5 \\
\hline Mediate Parallel System & 1,16 & 1,5 & 0,66 & 1,33 & 0,66 & 0,83 & 0,66 & 0,66 \\
\hline
\end{tabular}




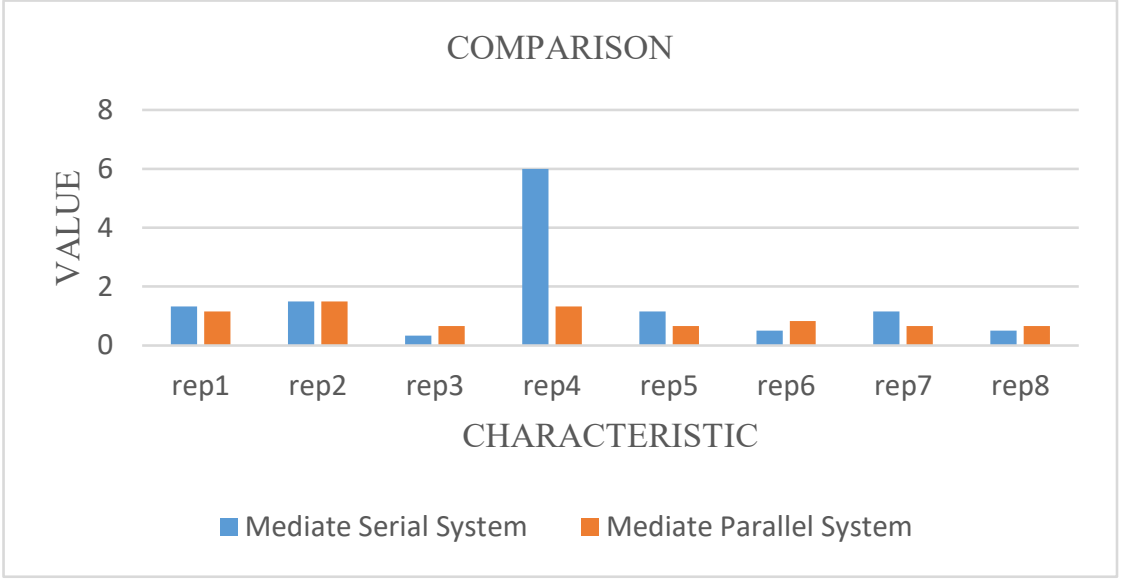

Fig. 12. Histogram comparing the failures of the two systems.

\section{Conclusions}

In the case of the series system, it was found that the first unit has a higher degree of wear due to prolonged use at a capacity of over $92 \%$, because it must heat the water to the first point controlled by the microcontroller. The second heat source operates in normal parameters and no aspects of excessive wear were observed.

From the calculations performed a significant increase in reliability can be observed in the case of parallel (cascade) heating systems using the same number and the same type of elements. The values obtained by analyzing equations (5) and (11) demonstrate the increase of the reliability of the parallel systems three times compared to the series systems.

It can be said that the design stage of reliability is one of the most important steps to obtain quality performance.

Experimentally, it can be seen that elements with high complexity, which also have low reliability, have a high failure rate. The average between the two systems is relatively uniform except for subsystems 4 and 7 which were designed and mounted in parallel.

This must be taken into account in the design of all elements and parts that have a low reliability.

In short, the main advantages of cascading heating units are: long-term investment efficiency; economical and highly efficient cascade communication solution through the use of intelligent control and monitoring devices; high energy efficiency compared to other heat sources; fully automated system; high modulation of the power of the heating system as a whole (for example, from 13 to $720 \mathrm{~kW}$ ); remote diagnosis and monitoring of units; respect for the environment; and last but not least high reliability.

\section{References}

1. C. Ștefănescu, Sisteme tolerante de defecte, (Ed. MatrixRom, Bucharest, 1999)

2. V.M. Cătuneanu, A. Mihalache, Reliability Fundamentals, (ELSEVIER, AnsterdamOxford-New York, Tokyo, 1989)

3. V. K. Ștefan, Evaluarea fiabilităţii sistemelor de calcul, (Ed. Universității din Oradea, Oradea, 2003)

4. T. Baron, Al. Isac-Maniu, Calitate și Fiabilitate, (Ed. Tehnică, București, 1998) 
5. https://derdomus.ru/ro/krysha-i-krovlya/shema-podklyucheniya-gazovyh-kotlov-vkaskad-kaskadnyi-montazh-otopitelnyh/ [Accessed 01.03.2021]

6. A.M. Alecusan, A. Dimitrescu, Innovation management: the past, present and future of the market, Studies in Business and Economics 11 Issue 3 140-149 (2016)

7. Yu.K. Mashkov, A.A. Gladenko, N.A. Prokudina, Increase of Reliability and Durability of Shaft Packing-off of Oil-transfer Pulser of the Main Oil Pipelines, Procedia Engineering 152 715-719 (2016)

8. A. Sohani, M.H. Shahverdian, and others, Selecting the best nanofluid type for A photovoltaic thermal (PV/T) system based on reliability, efficiency, energy, economic and environmental criteria, Journal of the Taiwan Institute of Chemical Engineers 120 (2021)

9. J. The, C.-M. Lai, Reliability impact of thedynamic thermal rating and battery energy storage systems on wild-integrated power networks, Sustainable Energy, Grids and Networks 20 (2019)

10. X. Liu, G. Zhu, T. Asiam, Y. Zhang, R. Mishra, The innovative design of air caps for improving the thermal efficiency of CFB boilers, Energy 221 (2021)

11. S. Gradziel Analysis of thermal and flow phenomena in natural circulation boiler evaporator, Energy 172 881-891 (2019) 